\title{
Educación para la Paz, Creatividad Atenta y Desarrollo Sostenible
}

\author{
Peace Education, Attentive Creativity and Sustainable \\ Development
}

\author{
Sonia París Albert * \\ Universitat Jaume I de Castellón, España
}

\begin{abstract}
Las sociedades actuales demandan un mayor compromiso ético, crítico y creativo, este texto reivindica un mayor cultivo de la educación para la paz, la cual ha de hacer posible, también, la reflexión sobre contenidos que van más allá de los currículums más clásicos y que atañen, por ejemplo, al desarrollo sostenible. En este sentido, se concibe la educación para la paz como una herramienta fundamental para la consecución de los Objetivos del Desarrollo Sostenible que se exponen en la Agenda 2030. Una educación para la paz que reclama, de manera muy especial, el cultivo de una creatividad atenta a la hora de abordar todas las cuestiones que afectan al desarrollo sostenible y, con ello, a la construcción, establecimiento y mantenimiento de paces creativas para un desarrollo sostenible. El carácter prioritario de la creatividad atenta en la educación para la paz se aborda, aquí también, desde la cotidianidad y la sencillez, y desde el reconocimiento a los rasgos de la intersubjetividad y performatividad como dos de sus atributos esenciales.
\end{abstract}

Descriptores: Educación formal; Paz; Educación alternativa; Creatividad; Desarrollo sostenible.

Current societies demand a greater commitment to Peace Education. Using pedagogical methodologies alternative to the classic narratives of teachers, with which students' active participation is promoted as well as their ethical, critical and creative thinking, this text claims a greater cultivation of Peace Education, which should also make possible the reflection on contents that go beyond the classic curricula and that are related, for example, to sustainable development. In this sense, Peace Education is conceived as a fundamental tool for the achievement of the Sustainable Development Goals that are set out in the Agenda 2030. A Peace Education that demands, in a very special way, the cultivation of an attentive creativity to approach the issues related to the sustainable development and, therefore, the construction, establishment and maintenance of creative peace(s) for a sustainable development. The priority of attentive creativity in Peace Education is approached, here also, from everydayness and simplicity, and from recognition to the features of intersubjectivity and performativity as two of its essential attributes.

Keywords: Formal education; Peace; Alternative education; Creativity; Sustainable development.

*Contacto: sparis@fis.uji.es

ISSN: 2254-3139

www.rinace.net/riejs/ revistas.uam.es/riejs
Recibido: $\quad 3$ de enero 2019

$1{ }^{\text {a }}$ Evaluación: 4 de febrero 2019

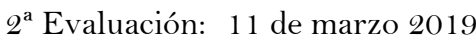

Aceptado: $\quad 20$ de abril 2019 


\section{Revisión de la literatura}

El papel de la educación en las sociedades actuales sigue siendo trascendental. De ahí que, cada vez, sean más los estudios que reflexionan sobre la misma, sobre su funcionamiento actual y sobre posibles alternativas a las tradicionales narrativas del profesorado, enfatizando, sobre todo, la necesidad de metodologías basadas en una participación más activa del estudiantado, en la importancia de promover el pensamiento ético, crítico y creativo, y en la posibilidad de crear espacios para trabajar contenidos que vayan más allá de los currículums clásicos y que permitan, por ejemplo, la presencia de una educación para la paz con carácter transversal.

Tanto la importancia de la educación, en general, como de la educación para la paz, en particular, se reconoce, claramente, en la Agenda 2030. En relación con ella, este texto propone cultivar la educación para la paz, de la mano de la creatividad atenta, en todos los niveles y contextos, muy especialmente, en los de la educación formal. Es decir, se plantea una educación para la paz que potencie la puesta en práctica de la creatividad atenta en lo que a la búsqueda de alternativas para la consecución de los Objetivos del Desarrollo Sostenible se refiere. Se trata, pues, de una educación para la paz que promueve formas creativas para lograr el desarrollo sostenible, y que se encamina hacia la construcción, establecimiento y mantenimiento de paces creativas para el desarrollo sostenible, a partir de un posicionamiento que hace pensar en la creatividad atenta desde la cotidianidad y la sencillez, así como teniendo muy en cuenta dos de sus atributos básicos: la intersubjetividad y la performatividad.

\subsection{La educación para la paz: Un medio esencial a la Agenda 2030 del Desarrollo Sostenible}

Del 25 al 27 de septiembre de 2015, los Jefes de Estado y de Gobierno y Altos Representantes, reunidos en la Sede de las Naciones Unidas en Nueva York, acordaron la Agenda 2030 para el Desarrollo Sostenible, pensada como un plan de acción que iba dirigido a la mejora de la calidad de vida de las personas y a la prosperidad del planeta, con la principal finalidad de fortalecer la paz universal (Naciones Unidas, 2015). De acuerdo con este propósito, la Agenda 2030 lista los que han sido llamados como los nuevos Objetivos del Desarrollo Sostenible de alcance mundial, ideados como medidas necesarias para la consecución del desarrollo sostenible, los cuales afrontan, con miras a su transformación en favor de una convivencia en paz, cuestiones relativas a los derechos humanos, la igualdad entre los géneros, el empoderamiento de las mujeres y las niñas, las desigualdades, el desempleo, la salud, los desastres naturales, la escalada de los conflictos violentos, las posturas extremistas, los terrorismos, las crisis humanitarias, el agotamiento de los recursos humanos y el cambio climático, entre otros. Toda una serie de cuestiones que se abordan en los 17 Objetivos del Desarrollo Sostenible que plantea la Agenda 2030, entre los que, ahora, me gustaría destacar, por la temática del estudio que aquí se presenta, el Objetivo 4, que señala "garantizar una educación inclusiva y equitativa de calidad y promover oportunidades de aprendizaje permanente para todos” (Naciones Unidas, 2015, p. 19), en cualquiera de los niveles de enseñanza. Objetivo que, sin ningún lugar a la duda, pone de manifiesto el reconocimiento que, una vez más, se da a la educación formal como motor de cambio social, siendo considerado, por consiguiente, el acceso a la misma en condiciones de igualdad, como una necesidad básica para todas las personas. 
El papel social de la educación formal se ha puesto, desde siempre, encima de la mesa. De ahí, que no hayan sido pocas las investigaciones que han debatido y dialogado sobre ella y sobre todo aquello que le concierne. En este sentido, por ejemplo, se pueden leer muchísimos trabajos que abordan las metodologías didácticas narrativas que, mayoritariamente, han colmado las aulas (Bourdieu y Passeron, 1967, 2001) y/o gran variedad de estudios que proponen otras pedagogías, mucho más centradas en el papel del diálogo entre el estudiantado y el profesorado como herramienta de aprendizaje (Greene, 2005). Es en esta última línea de trabajo que han ido viendo la luz investigaciones que influenciadas, muy especialmente, por la pedagogía libertaria de Freire (1970), aluden, sobre todo, a un más que necesario cambio en los roles que, tradicionalmente, las y los profesores y las y los estudiantes han tenido, de tal modo que ambos puedan empezar a ser interpretados como co-educadores en un proceso de aprendizaje y enseñanza en el que, tanto el estudiantado como el profesorado, harán oír su voz libre e igualitariamente, transformándose todos ellos en "sujetos del proceso en que crecen juntos y en el cual "los argumentos de la autoridad" ya no rigen” (Freire, 1970, p. 90), llegando a superar, así, la habitual "contradicción educador-educando" (Freire, 1970, p. 77) y percibiéndose, a sí mismos, como educadores y educandos al mismo tiempo. Se trata, pues, de propuestas alternativas que tienden hacia pedagogías mucho más dialógicas y reflexivas, y que fomentan una mayor participación activa y dinámica del estudiantado en el aula.

Si bien es cierto que estos modelos educativos alternativos a las tradicionales narrativas del profesorado son tendencia en la actualidad, hay que reconocer, también, que las pedagogías clásicas, basadas, sobre todo, en la figura de un profesorado que "hace comunicados y depósitos que los educandos, meras incidencias, reciben pacientemente, memorizan y repiten" (Freire, 1970, p. 76), siguen teniendo una gran presencia en nuestros días. Pedagogías clásicas que, además, siguen enfatizando "esencialmente, los saberes más académicos y racionales, las materias clásicas del currículum, la súper especialización, la división en compartimentos estancos del saber en materias o asignaturas, los métodos más discursivos o verbalistas..." (Dios Diz, 2013, p. 159), reduciendo, por consiguiente, a su mínima expresión, cualquier posible espacio para el diálogo y la reflexión sobre cuestiones ajenas a los contenidos del currículum más tradicional (París Albert, 2017, p. 70). Como consecuencia, muy poca cabida tiene aquí, por ejemplo, el trabajo sobre los contenidos expuestos en la Meta 4.7. del Objetivo 4 de la Agenda 2030 para el Desarrollo Sostenible, la cual reza del siguiente modo:

\section{De aquí a 2030, asegurar que todos los alumnos adquieran los conocimientos teóricos y prácticos necesarios para promover el desarrollo sostenible, entre otras cosas mediante la educación para el desarrollo sostenible y los estilos de vida sostenibles, los derechos humanos, la igualdad de género, la promoción de una cultura de paz y no violencia, la ciudadanía mundial y la valoración de la diversidad cultural y la contribución de la cultura al desarrollo sostenible. (Naciones Unidas, 2015, p. 20)}

La consecución de esta meta se podría hacer mucho más viable mediante la práctica de pedagogías alternativas a las tradicionales, las cuales, como se ha señalado más arriba, se centran, sobre todo, en los contenidos más clásicos del currículum. El éxito de esta meta requeriría, pues, pedagogías capaces de crear espacios para el diálogo entre el estudiantado y el profesorado y de potenciar, de esta forma, el pensamiento crítico, ético y creativo del estudiantado, no sólo sobre las materias tradicionales, sino, también, sobre todas aquellas otras cuestiones que afectan al bienestar de las personas y de la naturaleza, como, por ejemplo, son las que se indican en la Meta 4.7. del Objetivo 4 de la Agenda 2030, en favor de la construcción, establecimiento y mantenimiento de la paz y del desarrollo sostenible. 
Se trata de pedagogías, por lo tanto, capaces de producir un cambio revolucionario en la educación (Cabrera, 2018) y en las que, al mismo tiempo, es mucho más factible la incorporación transversal de una educación para la paz "global, crítica, emancipatoria, no sexista, solidaria, cooperativa, que evite la exclusión, transformadora..." (Dios Diz, 2013, pp. 158-159), al mismo tiempo que apta para sensibilizar en los "cuatro objetivos interactivos que comparten todos los elementos de la Educación para la Cultura de la Paz" (Tuvilla Rayo, 2004, p. 404), que son preparar para la no violencia, responsabilidad de los ciudadanos del mundo, igualdad de actitudes e investigación crítica de alternativas (pp. 404-405).

Así, pues, la puesta en práctica de la Agenda 2030 necesita del diálogo con la educación para la paz y de su aplicación en todos los niveles de la educación formal con el fin de poder llegar a sensibilizar, muy especialmente, en los Objetivos del Desarrollo Sostenible que la Agenda 2030 propone. Una educación para la paz en la que debe fomentarse, principalmente, una creatividad atenta, a la hora de buscar salidas creativas a las violencias y a las injusticias sociales para un desarrollo sostenible.

\section{La educación para la paz: El cultivo de la creatividad atenta para un desarrollo sostenible}

El fomento transversal de la educación para la paz en los centros de educación formal se entiende como un medio esencial a la Agenda 2030, ya que, en efecto, es una herramienta con la que se potencia el conocimiento teórico y práctico de las cuestiones que afectan a la paz y, con ello, a los Objetivos del Desarrollo Sostenible, favoreciendo, así mismo, en su sensibilización. No obstante, como se ha indicado al final del apartado anterior, lo que este trabajo propone es una educación para la paz que promueva una creatividad atenta. Esto es, una educación para la paz creativa que ha de impulsar el cultivo de salidas pacíficas, creativas y con cuidado a las diferentes formas de violencia, para la consecución de un desarrollo sostenible.

No cabe duda de que todo el mundo habla de la creatividad actualmente y de que aparece en los discursos de todos los ámbitos, proponiéndose, continuamente, como herramienta para la mejora en el desarrollo de cualquier acción. Sin embargo, la pregunta que, aquí, surge es: ¿Cómo se entiende la creatividad en los discursos actuales? Es evidente que el futuro de la humanidad va unido a la creatividad, muy especialmente, por las maneras cómo sus resultados "enriquecen la cultura y, de ese modo, mejoran indirectamente la calidad de nuestras vidas" (Csikszentmihalyi, 1998, pp. 25-26). A pesar de ello, ni que decir tiene que, según en qué principios se basen esa mejora y enriquecimiento, se conceptualizará y, por consiguiente, se ejercerá la creatividad. A lo que me refiero es a que la mayoría de las sociedades occidentales han deshumanizado la creatividad, poniéndola al servicio de unos criterios de utilidad productiva que miden tanto el emprendedorismo como la innovación de acuerdo con su rentabilidad económica. Así, actualmente, impera, por encima de todo, la idea de una creatividad mercantilista, definida como una herramienta capaz de producir ideas en un mundo que medirá su utilidad y necesidad en función de los beneficios económicos que aporte al sistema financiero. Es con este mismo sentido con el que se da, por ejemplo, cuando al sistema universitario se le reclama que capacite al estudiantado, en "un mundo totalitario del trabajo" (Pieper, 1970, p. 23), para liderar proyectos que mejoren, por ejemplo, el bienestar de las personas y/o el desarrollo 
sostenible, aunque sea desde una visión reduccionista de ese bienestar y/o desarrollo, al medirlos y valorarlos con criterios, puramente, economicistas.

Frente a esta noción de creatividad mercantilista, se quiere poner encima de la mesa, aquí, la reivindicación hacia la necesaria humanización de la creatividad, poniendo en tela de juicio, para ello, también, el concepto de utilidad productiva y económica en el que este modo de ver la creatividad se fundamenta (Garcés, 2015). De esta manera y de acuerdo con el oxímoron La utilidad de lo inútil usado por Ordine en el título de uno de sus libros, se hace, absolutamente necesario, subvertir el término de utilidad, mostrando que lo realmente útil para la humanidad es aquello que hoy se cree inútil (Ordine, 2013). Esto es, todos aquellos saberes que, por no corresponderse con los criterios economicistas actuales, tienen, justamente, su utilidad en su aparente inutilidad (París Albert, 2018a). Sólo, desde este punto de vista, es como la visión mercantilista de la creatividad da paso a una creatividad atenta que, caracterizada por ser dispuesta, resuelta y decidida, viva, enérgica y dinámica, e incansable y laboriosa, humaniza la creatividad, al ser mucho más cuidadosa con los asuntos humanos, así como con la pluralidad y con la diversidad, en la búsqueda de nuevas, más y mejores ideas, que no tomarán los principios económicos como únicos criterios de medida del bienestar, de la calidad de vida de las personas y del desarrollo sostenible. A diferencia de ello, el punto de partida de la creatividad atenta, está en su predisposición hacia el cuidado (Comins Mingol, 2018), por lo que toma muy en cuenta al ser humano en sí mismo y en interacción con todo aquello que le rodea, lo que le lleva a valorar la particularidad de cada caso en la búsqueda de salidas a los problemas individuales y sociales, y a cultivarse de forma respetuosa, educada, considerada y cordial. Se trata, por lo tanto, de una creatividad atenta que imagina alternativas cuidadosas con las que afrontar las situaciones diarias y, también, las situaciones de injusticia, las desigualdades sociales, las crisis ambientales, el desarrollo sostenible, etc. Una creatividad atenta que, de hecho, se defiende en estas páginas, debe impulsarse en el marco de una educación para la paz aplicable en todos los contextos educativos, muy especialmente en los de la educación formal, como recurso para ser capaces de hacernos las paces de formas diferentes a las habituales, yendo más allá de lo esperado. Una creatividad atenta que pone su énfasis, también, en una resignificación del valor del tiempo (Comins Mingol, 2018, p. 88), según la que se presta mucha más atención a aquello que se hace en cada momento y que permite dedicar los minutos necesarios al desarrollo de cada acción. De hecho, ni la curiosidad ni el cuidado, ni el reconocimiento ni la solidaridad, ni la cooperación ni el amor, con los que esta creatividad atenta se mueve, pueden ser puestos en práctica con prisas, sino más bien, con la calma necesaria que ha de hacer pensar, tranquilamente, en lo que se hace y en el cómo se hace, y valorar todas las posibles alternativas, escapando de lo habitual y tomando en consideración, ante todo, a las personas y a su relación con el entorno. Ciertamente, es importante señalar que sólo cuando se tiene en cuenta esta manera atenta de entender la creatividad, es cuando se observa que la creatividad es una asignatura pendiente (Barrena, 2007), que no ha de contraponerse con la razón, sino, más bien, conceptualizarse como una de sus características esenciales, "resultado de diversos procesos mentales que se usan para generar y desarrollar nuevas ideas" (Barrena, 2007, p. 28).

Desde la propuesta del cultivo de la creatividad atenta en la educación para la paz, la creatividad puede verse, entonces, como una competencia (Marina y Marina, 2013) del pensamiento humano, como una capacidad en el sentido de Max-Neef (2000), junto con la capacidad del pensamiento crítico y ético (Sátiro, 2002, p. 145), y como una procesadora 
de la experiencia que da lugar a más, nuevas y mejores ideas (Sátiro, 2011a, 2011b) basadas en el cuidado (Comins Mingol, 2018), gracias a su fluidez, flexibilidad, originalidad y elaboración (Sátiro, 2005, p. 47). Tanto es así que, siguiendo a Lipman, Sátiro defiende la idea según la que "un pensamiento es más creativo a medida que es más flexible, fluido, original y capaz de elaborar todo aquello que procesa” (Sátiro, 2005, p. 47). Características, estas últimas, que delimitan al pensamiento creativo y que favorecen la presencia, en mayor o menor medida, de la creatividad atenta en todas las personas.

La creatividad atenta es percibida, por consiguiente, como el motor que humaniza a la creatividad y como absolutamente favorable a la búsqueda de alternativas creativas y cuidadosas, en el marco de una educación para la paz que trabajará sobre los principios expuestos en la Agenda 2030 con el fin de impulsar conocimientos y acciones para el cultivo de cada uno de los objetivos que en ella se listan, logrando, así y al mismo tiempo, un desarrollo sostenible creativo y con cuidado.

\section{La creatividad atenta: Hacia las paces creativas para el desarrollo sostenible}

La estimulación de la creatividad atenta mediante la educación para la paz se convierte, tal y como se viene señalando en las páginas anteriores, en una tarea necesaria en las sociedades actuales, si lo que se pretende es ofrecer más oportunidades para atreverse a ser, pensar y actuar de una manera alternativa, creativa y atenta, al encarar no sólo las situaciones diarias, sino, también, los escenarios de conflicto, las injusticias sociales, las desigualdades y el desarrollo sostenible, haciéndonos las paces (Martínez Guzmán, 2001). Se trata, pues, de promover, a través de la educación para la paz, la creatividad atenta como el hábito que ha de guiar nuestro actuar, también, en lo que a la construcción, establecimiento y mantenimiento de la paz se refiere, así como en lo que atañe a la consecución de los Objetivos del Desarrollo Sostenible expuestos en la Agenda 2030, ya que no cabe duda de que los contextos de violencia directa, cultural y estructural requieren, igualmente, escaparse de la rutina a través de miradas inusuales y originales, que sin dejar de ser creativas y atentas, han de hacer posible imaginar y dibujar salidas cuidadosas frente al terror, la crueldad, las injusticias, la pobreza y las desigualdades, siempre en favor de una convivencia en paz y de un desarrollo sostenible. Tanto es así que García González (2014, p. 4) insiste en que "estamos obligados a ser buscadores de paz y encontrar rumbos hacia los cuales ir, repensando la realidad frente a nosotros para indagar alternativas posibles desde una imaginación de carácter ético" (p. 4).

De acuerdo con lo anterior, el impulso de la creatividad atenta desde la educación para la paz pasa a ser una pieza imprescindible para la consecución de los Objetivos del Desarrollo Sostenible y, con ello, para hacernos las paces. Más aún, si tenemos en cuenta que el desarrollo sostenible y, por lo tanto el trabajo por la paz, implica una obra creativa de cuidar las necesidades vitales básicas mediante acciones y actos de habla que, en el marco de la pluralidad y el estar juntos de los seres humanos, son capaces de hacer lo que parece improbable (Martínez Guzmán, 2018, p. 23), a través de la "creatividad como motor de desarrollo [...] para mejorar la calidad de vida en general, reduciendo la pobreza e incidiendo en un mayor desarrollo" (Sátiro, 2009, p. 98), y permitiendo mantener, así, el "sueño de cambiar la realidad social" (Sátiro, 2009, p. 97). Obra creativa que da lugar, entonces, a unas paces creativas para el desarrollo sostenible, las cuales se mueven, 
fundamentalmente, por la curiosidad, el interés y el entusiasmo para emprender la aventura de arriesgar a la hora de afrontar, creativa y atentamente, las situaciones de conflicto, las injusticias sociales, la pobreza y el desarrollo sostenible en favor de la convivencia en paz (París Albert, 2018b). Unas paces creativas para el desarrollo sostenible que hacen replantear "algunos supuestos y reposicionarlos intentando sortearlos y emplazar a la paz -como objetivo a lograr- como un nuevo escenario susceptible de convertirse en realidad" (García González, 2014, p. 8) porque no cabe duda de que "la búsqueda de lo imaginable y de lo inimaginable, de lo inesperado y de aquello que emerge de la creatividad, es lo que podrá dar luces para concebir y favorecer proyectos de paz” (García González, 2014, p. 17). Para ello, en efecto, la construcción, establecimiento y mantenimiento de las paces creativas para el desarrollo sostenible necesita de narraciones creativas y cuidadosas que, incluyendo miradas apreciativas y estéticas con las que "apreciar lo bello para pensar nuevos caminos" (Ospina-Ramírez y Ospina-Alvarado, 2017, p. 182), tomarán muy en consideración los deseos y los sueños de las personas afectadas por los contextos de violencia directa, cultural y estructural, al tiempo que se esforzarán por aproximarse, todo lo más que se pueda, a ellos. De este modo, las paces creativas para el desarrollo sostenible se guiarán por narraciones creativas y atentas que no obviarán, en modo alguno, las ilusiones y las esperanzas de los sujetos durante los procesos de paz y el trabajo hacia la consecución de los Objetivos del Desarrollo Sostenible. Sujetos que, además, actuarán "leyendo la historia con todas sus implicaciones, pero manteniendo una mirada apreciativa que les ayude a reconocer los momentos y las situaciones que les han permitido alejarse de la violencia y los aprendizajes, cambios y potencias que se han generado en ellas, ellos y sus familias para empezar a caminar por nuevos caminos" (Ospina-Ramírez y Ospina-Alvarado, 2017, p. 183).

Las paces creativas para el desarrollo sostenible, de la mano de la estimulación de la creatividad atenta desde la educación para la paz, permitirán, entonces, reflexiones inusitadas y fantasiosas sobre los contenidos expuestos en la Agenda 2030 que, teniendo muy en cuenta el reconocimiento a la complejidad y pluralidad humana y a la diversidad de lugares en el mundo, romperán "con los paradigmas" (Ospina-Ramírez y OspinaAlvarado, 2017, p. 181), y crearán alternativas para la consecución de los Objetivos del Desarrollo Sostenible, basadas en la imaginación y en las siguientes competencias pacíficas (París Albert, 2018b, p. 174):

1. La competencia para comunicarnos creativamente, considerando a todas las partes en términos de igualdad y dando el mismo valor a todas las voces implicadas en el diálogo, para que todas las personas tengan la oportunidad de expresarse y de ser escuchadas de manera empática, sin juicios previos y desde una perspectiva cuidadosa, comprensiva, ética, crítica y creativa.

2. La competencia para reconocernos mutuamente en nuestra integridad física como sujetos con igualdad de derechos y deberes, que podemos tener distintas formas de ver y entender la vida. Se trata de reconocernos cuidadosamente unas personas a las otras, asumiendo nuestras diferencias, comprendiéndolas y dándoles valor desde una postura creativa.

3. La competencia para buscar acuerdos creativos basados en la cooperación, a través de los que se pueda ir más allá del simple querer ganar y se puedan adoptar otros posicionamientos más complejos, que lleven a esforzarse para intentar encontrar salidas igual de beneficiosas para todas las personas, superando, así, la perspectiva más individualista para situarse en otra mucho más colaborativa y atenta. 
4. La competencia para percibir las cosas desde distintos puntos de vista y con creatividad, obviando los estereotipos, restando fuerza a nuestros puntos de vista para sumársela a los de las otras personas y tratando de indagar otras maneras de hacer las cosas, que hagan posible la comprensión cuidadosa de la complejidad humana y de la diversidad de lugares que hay en el mundo.

5. La competencia para ejercer nuestros poderes de una manera integrativa y creativa, que evite la jerarquización humana y la subordinación de unas personas bajo el dominio de otras. Poderes que faciliten la concertación atenta de acuerdos y que sean muy conscientes de la fragilidad y de la vulnerabilidad humana.

6. La competencia para responsabilizarnos de nuestras acciones y de nuestras palabras $y$ de ofrecer respuestas creativas y cuidadosas para afrontar sus consecuencias.

7. La competencia para hacer fluir los sentimientos, sacándolos desde dentro hacia afuera y regulándolos de una manera creativa y atenta, para no dejarnos llevar por los más propicios a la violencia y sí, en cambio, por aquellos más cercanos a la convivencia en paz.

8. La competencia para indignarnos con situaciones que afectan desfavorablemente a la vida de otras personas y para comprometernos en la búsqueda de salidas creativas y cuidadosas en favor de su bienestar individual y social, trabajando por reducir al máximo cualquier tipo de injusticia desigualdad.

\subsection{La educación para la paz: Una reivindicación a la cotidianidad y a la sencillez en la creatividad atenta para el desarrollo sostenible}

Cabe tener en cuenta que el fomento de la creatividad atenta para el desarrollo sostenible y, con ello, para la construcción, establecimiento y mantenimiento de la paz no debe dejarse al amparo, únicamente, de situaciones extraordinarias y de personas con posiciones notables de poder porque, al hacerlo de este modo, lo que puede suceder es que se dé una visión muy limitada de la creatividad, la cual la relacionará, sobre todo, con "individuos que, como Leonardo, Edison, Picasso o Einstein, han cambiado nuestra cultura en algún aspecto importante" (Csikszentmihalyi, 1998, p. 44). Es decir, con sujetos que hacen grandes obras que generan magníficos productos y que tienen, al mismo tiempo, en su personalidad, toda una serie de rasgos antitéticos, que pueden mostrarse del siguiente modo (Csikszentmihalyi, 1998, p. 80): tanto en su energía física como en sus tiempos para el reposo y la calma; en su ingenuidad como en su viveza; en su tendencia hacia la responsabilidad y la disciplina como hacia la irresponsabilidad y lo lúdico; en su imaginación y fantasía como en su arraigado sentido de la realidad; en su extroversión como en su introversión; en su humildad como en su orgullo; en su rebeldía e independencia como en su talante conservador y tradicional; en su pasión por el trabajo como en su objetividad respecto al mismo; en su sensibilidad hacia el dolor como hacia el placer; y en su habilidad para escapar de los más generalizados estereotipos de género, soliendo ser las mujeres más duras y dominantes y los hombres más sensibles y menos agresivos. Se trata ésta, pues, de una cosmovisión tan delimitada de la creatividad, que no sólo se vincula con aquellas personas que tienen unos rasgos muy concretos, sino que, también, ofrece una posición muy especial a los entornos, hasta el punto de señalar que "escoger el entorno erróneo probablemente dificultará el despliegue de la creatividad" (Csikszentmihalyi, 1998, p. 164) y de distinguir, claramente, cuáles son los ambientes creativos de los que no, siempre en función del periodo en el que se encuentra el proceso creativo, pues parte de la percepción de que, "aunque los entornos novedosos y bellos pueden catalizar el momento de la intuición, las demás fases del proceso creativo- como la preparación y la evaluación- parecen beneficiarse más de escenarios conocidos y cómodos, 
aun cuando estos a menudo no sean mejores que un desván” (Csikszentmihalyi, 1998, p. 170).

Más allá de esta concepción tan ceñida, que prioriza la personalidad creativa, los entornos, el proceso y los productos, la perspectiva, desde la que se está trabajando la creatividad atenta para el desarrollo sostenible en estas páginas, supone revalorizar lo cotidiano en la educación para la paz, como cualidad que refiere a aquello que es diario, corriente, ordinario, habitual, regular, periódico o usual (Toro, 2017, pp. 24-25). En este sentido, implica la reivindicación del día a día, de las situaciones que, por ser cercanas a las personas, por vivirlas y ser parte de nuestras experiencias vitales, son mucho más comprensibles para cada una de nosotras. Esto es, entraña la reivindicación de una cotidianidad (Marina y Marina, 2013) que, en muchos casos, ya se suele afrontar de manera creativa y atenta y cuya revalorización beneficia, sobremanera, el desarrollo de un ser, pensar y actuar creativo como hábito para avanzar, también, desde los niveles micro a los de carácter más macro, sirviendo de paradigma, así, a la hora de abordar, por ejemplo, los Objetivos del Desarrollo Sostenible expuestos en la Agenda 2030 y, con ello, la construcción, establecimiento y mantenimiento de las paces creativas para el desarrollo sostenible. En efecto, son muchos los aprendizajes que se pueden extraer de las maneras con las que afrontamos creativa y atentamente nuestra vida diaria; con las que resolvemos encajar las piezas del puzle de nuestras vivencias cotidianas; con las que gestionamos alternativamente y con cuidado nuestros conflictos, así como con las que nos esforzamos por lograr nuestro bienestar con las y los demás y con la naturaleza. Desde esta perspectiva, entonces y como se ha venido defendiendo en este texto, el cultivo que, desde la educación para la paz, se hará de la creatividad atenta para el desarrollo sostenible no está hecho sólo para grandes genios, sino, también, para las "personas que expresan pensamientos inusitados, que son interesantes y estimulantes" (Csikszentmihalyi, 1998, p. 43), para las "que experimentan el mundo de maneras novedosas y originales" (Csikszentmihalyi, 1998, p. 43) y para cualquiera de nosotras y nosotros, quienes, desde nuestros propios contextos, teniendo en cuenta nuestras circunstancias y lo que nos rodea, somos capaces de imaginar maneras alternativas de relacionarnos con cuidado, cordialidad y empatía, visualizando los diferentes puntos de vista y desarrollando un pensamiento crítico y ético que nos ayude a idear salidas para nuestros quehaceres diarios, en favor de un desarrollo sostenible y de una convivencia en paz. En este sentido, dice Barrena (2007, p. 10) que como el ser humano "es creativo por naturaleza, tiende a crecer, a desarrollarse de maneras que no le vienen dadas, a manifestarse libremente a través de la ciencia, del arte o simplemente a través de las acciones que desarrolla en su vida cotidiana”. Ello, evidentemente, estemos las personas en el lugar en el que estemos, ya sea desde la posición privilegiada de quien tiene una familia, amigos y un trabajo hasta la de quien vive en escenarios cargados de violencia, pues, como se ha señalado anteriormente, también, en estos contextos, el cultivo de la creatividad atenta puede jugar un papel trascendental en la construcción de las paces creativas para el desarrollo sostenible.

Lo cotidiano se ha de poner en diálogo, también, con la reivindicación que, desde la educación para la paz, debe hacerse de la sencillez, entendiendo a ésta como contraparte de la complejidad (Lederach, 2007; Muñoz Muñoz y Molina Rueda, 2010). Ciertamente, no hay duda de que la humanidad es compleja (París Albert, 2015), de que la vida en general es compleja y, por este motivo, las personas solemos creer que, también, las soluciones más eficaces para afrontar nuestros problemas, los conflictos sociales, el desarrollo sostenible y la paz deben estar basadas en la complejidad. Frente a ello, este 
texto propone recuperar el valor de lo sencillo como base de la complejidad (Lederach, 2007, p. 65), de tal modo que se vea que, para comprender la complejidad de cualquier cosa o acción, hay que rescatar sus esencias, es decir, aquellas cuestiones básicas que la definen y que la hacen ser lo que es, lo que supone, por consiguiente, avanzar hacia y desde la sencillez. Se trata, pues, de no percibir la relación entre lo sencillo y lo complejo como la de dos polos antagónicos, sino, más bien, como la de un proceso que va de lo complejo a lo sencillo y de lo sencillo a lo complejo (Panikkar, 1993, p. 10), dividiendo cualquier cosa o acción en todas sus partes básicas con el fin de aprehenderlas, en primer lugar, individualmente $\mathrm{y}$, después, en su conjunto, a lo que hay que añadir, también, las interrelaciones, incertidumbres y azares que forman un sistema y las características emergentes que son distintas a las de sus partes (Morin, 1998). Esto es, se trata de armonizar la complejidad captándola desde la sencillez (Panikkar, 1993, p. 10).

A esta relación entre la sencillez y la complejidad llega, también, Lederach en su libro "La imaginación moral. El arte y el alma de la construcción de la paz" de 2007 tras detenerse a mirar el movimiento de las bandadas de pájaros e intentar comprenderlo. Para Lederach, se hace muy difícil creer que la mente humana pueda entender este movimiento, tan complejo y perfecto, a no ser que no se intente analizar en su conjunto, como un todo único, sino a partir de cada una de sus piezas individuales. A través de esta percepción, es como el autor concluye que "la clave de la complejidad es encontrar la elegante belleza de la sencillez" (Lederach, 2007, p. 69) y, de acuerdo con ello, desarrolla su investigación por la paz, planteándose la siguiente pregunta: “ ¿cuáles son los tres o cuatro elementos absolutamente básicos de que consta la construcción de la paz?”, (Lederach, 2007, p. 68). Aquí y por la temática de este estudio, la pregunta a plantear sería: ¿cuáles son las «esencias» fundamentales (Lederach, 2007) que cabe tener en cuenta desde la educación para la paz, al adentrarnos en la reflexión sobre los Objetivos del Desarrollo Sostenible de la Agenda 2030, buscando salidas creativas y cuidadosas propicias a las paces creativas para el desarrollo sostenible?

De acuerdo con las ideas anteriores, se puede afirmar, entonces, que la educación para la paz debe basar el cultivo de la creatividad atenta en una reivindicación de la cotidianidad y de la sencillez, a la hora de abordar y sensibilizar en los objetivos de la Agenda 2030, siempre en favor de la construcción, establecimiento y mantenimiento de las paces creativas para el desarrollo sostenible.

\section{Educación para la paz: La intersubjetividad y la performatividad como dos atributos de la creatividad atenta para el desarrollo sostenible}

Así como, en el apartado anterior, se ha defendido la importancia de reivindicar, desde la educación para la paz, la cotidianidad y la sencillez en la creatividad atenta para el desarrollo sostenible, se quiere enfatizar, ahora, dos de sus propiedades distintivas, la intersubjetividad y la performatividad, las cuales, sin ninguna duda también, deben ser trabajadas en la educación para la paz. Propiedades que llevan a resaltar la idea de una creatividad atenta intersubjetiva y performativa para la consecución de los Objetivos del Desarrollo Sostenible de la Agenda 2030, con la que se pretende poner encima de la mesa el importante papel que juegan, para su práctica, las maneras cómo se desenvuelven las relaciones entre las personas. Tanto es así que cabe decir que, cuando se habla de la 
intersubjetividad como atributo esencial de la creatividad atenta para el desarrollo sostenible, se hace alusión, principalmente, a las influencias que las otras y los otros pueden tener sobre nuestra creatividad. En efecto, la creatividad no sólo depende de la personalidad creativa, sino, también y, sobre todo, de las maneras cómo hemos aprendido a ser personas creativas y atentas en nuestros contextos, con nuestras familias, amigos y conocidos, así como de cualquier influencia externa que hemos ido recibiendo para el desarrollo de nuestra creatividad atenta y de los modos en los que ha ido siendo reconocida. En este sentido, por ejemplo, no resulta extraño encontrar sujetos que tienen una gran personalidad creativa, pero que, en cambio, no la han desplegado lo suficiente, al no haber sido educados en ella; al no habérseles potenciado desde sus entornos. Esta es una de las razones por las que, en este texto, se ha destacado el papel de la educación, especialmente, de la educación formal en lo que al devenir de la creatividad atenta para el desarrollo sostenible afecta, pues no cabe duda de que puede ser educada y de que, en función de las metodologías pedagógicas utilizadas, se puede promover más o menos, tanto en sentido positivo como negativo. Por consiguiente, ni que decir tiene que la creatividad atenta para el desarrollo sostenible es intersubjetiva, al depender su puesta en práctica de los contextos en los que vive cada persona y de las formas cómo se potencia en función de cómo se concibe el ser creativo en cada uno de estos escenarios. En definitiva, de las maneras cómo se aprende a ser creativo y de cómo se influye o se reconoce la creatividad atenta por las y los demás según cada contexto.

El hecho de resaltar el carácter intersubjetivo de la creatividad atenta para el desarrollo sostenible nos ayuda a quitar, por consiguiente, todo su peso de la personalidad creativa. Respecto a ello, es importante recordar que los estudios más clásicos sobre la creatividad siempre han prestado una atención especial a la personalidad del sujeto, poniendo el énfasis en si reunía las características necesarias para ser identificado como un persona creativa o no. Esta tendencia, sin embargo, ha ido cambiando con el paso del tiempo y así, por ejemplo, Csikszentmihalyi (1998) ha sido uno de los autores que ha puesto todo su empeño en diseñar una concepción de la creatividad alejada de esa corriente. Para ello, se ha movido en el marco de la Teoría de Sistemas, según la que no es sólo la personalidad del individuo lo que se debe tratar a la hora de hablar de la creatividad, sino, también y muy por encima de todo, su vinculación con lo que él viene a llamar como el campo y el ámbito. Así, mientras que con el término campo se refiere a un conjunto de reglas y procedimientos simbólicos, con el de ámbito "incluye a todos los individuos que actúan como guardianes de las puertas que dan acceso al campo" (Csikszentmihalyi, 1998, p. 46). Ello lo que le lleva a decir que "puesto que la creatividad está constituida conjuntamente por la interacción entre campo, ámbito y persona, el rasgo de la creatividad personal puede ayudar a generar la novedad que modifique dicho campo, pero no es una condición suficiente ni necesaria para ello" (p. 47).

Es bien cierto que Csikszentmihalyi (1998) aplica su teoría a una visión muy limitada de la creatividad, la cual se vincula, exclusivamente, con las personas que se conocen como los grandes genios creativos. A diferencia de ello, como se ha señalado previamente en este texto, se quiere extrapolar la posibilidad de la creatividad atenta para el desarrollo sostenible a un nivel más generalizado, el cual abarca la oportunidad de entender a todas las personas como individuos creativos, al tiempo que nos lleva a poder decir que la creatividad atenta para el desarrollo sostenible es intersubjetiva porque no depende sólo de una o de uno mismo, sino, sobre todo, de las maneras cómo dicha creatividad se ejerce de acuerdo con los modos cómo se ha aprendido a hacerlo en un contexto determinado, de 
las influencias que recibe en ese entorno y de las formas cómo se reconoce por las y los demás. Hablamos, por consiguiente, de un carácter social de la creatividad atenta para el desarrollo sostenible que implica reconocer, al mismo tiempo, su talante comunicativo y dialógico. De hecho, la creatividad atenta para el desarrollo sostenible requiere que las personas establezcamos diálogos a partir de los que nos comuniquemos sobre las maneras en las que entendemos la creatividad y sus consecuencias; diálogos a través de los que poder identificar qué es ser creativas y atentas en cada entorno; qué es lo que cada escenario demanda cómo creativo en lo que a la consecución de la Agenda 2030 se refiere, lo que se quiere, lo que se necesita y cómo se solicita. Ciertamente, se trata, entonces, de una visión social y relacional de la creatividad atenta para el desarrollo sostenible que pone el énfasis en la interacción entre las personas a través de su carácter intersubjetivo, y que debe ser trabajada en la educación para la paz.

El segundo atributo esencial de la creatividad atenta para el desarrollo sostenible que se quiere destacar en este trabajo es el que hace referencia a su performatividad (Martínez Guzmán, 2001). Con el concepto de performativo se quiere decir que las personas podemos pedirnos cuentas por las formas en las que somos o dejamos de ser creativas. Por ello, el carácter performativo de la creatividad atenta para el desarrollo sostenible supone, entonces, la oportunidad para pedirnos cuentas por las maneras en las que nos relacionamos creativa y atentamente, así como por las consecuencias que se derivan de estas relaciones. Una concepción de la creatividad atenta que, como se puede observar, viene estando muy influenciada desde la filosofía, por ejemplo, por la escuela de la Teoría de los Actos de habla de Austin (1971). Aplicado a los actos de habla, Austin afirma que todo decir es un hacer y que, por lo tanto, podemos pedirnos cuentas por las maneras en las que nos decimos o nos callamos las cosas, así como de sus efectos sobre las y los demás. En esta misma línea, este estudio lo que propone es comprender las acciones ejercidas con creatividad atenta como cualquier otra acción, por la que, también, podemos pedirnos cuentas por ellas. Cada sujeto es responsable de las acciones que hace en relación con los Objetivos del Desarrollo Sostenible y de si sus acciones son creativas y atentas o no, por lo que siempre podemos pedirnos cuentas y exigirnos tanto más creatividad como alguna forma concreta de entenderla. Es este el motivo por el que, entonces, aquí se ha considerado tan necesario cultivar, desde la educación para la paz y para la consecución de la Agenda 2030 del desarrollo sostenible, una creatividad mucho más atenta que, revalorizada desde la sencillez y lo cotidiano, puede ser cultivada en todos los sujetos y contextos, también en aquellos que, por ser violentos, se esfuerzan asiduamente por la construcción, establecimiento y mantenimiento de paces creativas para el desarrollo sostenible.

\section{Conclusiones}

A continuación, se señalan las principales conclusiones alcanzadas en estas páginas:

- A pesar de que se reconocen los grandes esfuerzos que, en los últimos tiempos, se están haciendo por usar metodologías distintas a las clásicas narrativas del profesorado en la educación formal, este artículo enfatiza la necesidad de seguir trabajando en esta línea y de impulsar, así, metodologías alternativas que den un papel más activo al estudiantado, así como la posibilidad de trabajar desde la perspectiva de una educación para la paz en las aulas. En este sentido, la pedagogía libertaria de Freire se convierte en una propuesta útil, ya que provoca 
una subversión de roles entre lo que, tradicionalmente, se ha entendido como el estudiantado y el profesorado. Tanto es así que, para la pedagogía libertaria, todos los agentes implicados en el proceso de aprendizaje-enseñanza educan y son educados, poniendo en valor, por lo tanto, todas las voces. Se trata, por consiguiente, de una pedagogía muy propicia, también, al desarrollo del pensamiento crítico, ético y creativo, pues da al estudiantado la posibilidad de reflexionar y argumentar desde diferentes puntos de vista y perspectivas.

- La educación para la paz se cree que es una herramienta esencial para la consecución de los Objetivos del Desarrollo Sostenible de la Agenda 2030. Una educación para la paz que, en este estudio, se ha defendido debe cultivar, sobre todo, la creatividad atenta. La tendencia debería ser, entonces, la de ir dando, cada vez, más cabida a la educación para la paz en los contextos de educación formal con la finalidad de impulsar la reflexión sobre las cuestiones que le atañen, como puede ser la consecución de la Agenda 2030. Una reflexión que debería hacerse desde la creatividad atenta, por lo que se hace necesario trabajar con creatividad y para la creatividad en las aulas.

- El impulso de la creatividad atenta en la educación para la paz hace posible la construcción, establecimiento y mantenimiento de paces creativas para el desarrollo sostenible. Unas paces creativas que ponen el énfasis en la búsqueda de alternativas creativas y atentas y que ponen encima de la mesa la pluralidad de personas, de culturas y de maneras en las que podemos hacernos las paces.

- La educación para la paz debe resaltar la cotidianidad y la sencillez en la creatividad atenta para el desarrollo sostenible, así como la intersubjetividad y la performatividad como dos de sus atributos esenciales. Todo ello, desde la perspectiva de la paz positiva, con la que se asume tanto la complejidad como la conflictividad de la naturaleza humana.

\section{Referencias}

Austin, J. L. (1971). Palabras y acciones. Cómo hacer cosas con palabras. Buenos Aires: Paidós.

Barrena, S. (2007). La razón creativa. Crecimiento y finalidad del ser humano según S. S. Peirce. Madrid: Ediciones Rialp.

Bourdieu, P. y Passeron, J. C. (1967). Los estudiantes y la cultura. Barcelona: Editorial Labor.

Bourdieu, P. y Passeron, J. C. (2001). La reproducción. Elementos para una teoría del sistema de enseñanza. Madrid: Editorial Popular.

Cabrera, J. (2018). La creatividad y su revolución para la mejora educativa y la justicia social. Revista Internacional de Educación para la Justicia Social, 7(2), 7-10.

Comins Mingol, I. (2018). De la parálisis a la reconexión: por una fenomenología y una creatividad pacifistas. En S. París Albert y S. Herrero Rico (eds.), El quehacer creativo. Un desafio para nuestra cotidianidad (pp. 85-98). Madrid: Dykinson. https://doi.org/10.2307/j.ctv301f3z.9

Csikszentmihalyi, M. (1998). Creatividad. El fluir y la psicología del descubrimiento y la invención. Barcelona: Paidós.

Dios Diz, M. (2013). El currículum de la noviolencia en la educación para la paz. En I. Comins Mingol y F. A. Muñoz (Eds.), Filosofías y praxis de la paz (pp. 141-164). Barcelona: Icaria.

Freire, P. (1970). Pedagogía del oprimido. Buenos Aires: Siglo XX1. 
Galtung, J. (1969). Violence, peace and peace research. Journal of Peace Research, 6(3), 167-191. https://doi.org/10.1177/002234336900600301

Garcés, M. (2015). Filosofía inacabada. Barcelona: Galaxia Gutemberg.

García González, D. E. (2014). Hacia una prospectiva de paz a partir del realismo de la violencia: Una construcción desde la imaginación ética. En E. D. García González (Ed.), Trascender la violencia. Críticas y propuestas interdisciplinarias para construir la paz (pp. 3-30). Ciudad de México: Editorial Porrúa.

Greene, M. (2005). Liberar la imaginación: Ensayos sobre educación, arte y cambio social. Barcelona: Graó.

Lederach, J. P. (2007). La imaginación moral. El arte y el alma de la construcción de la paz. Bilbao: Bakeaz.

Marina, J. A. y Marina, E. (2013). El aprendizaje de la creatividad. Barcelona: Ariel.

Martínez Guzmán, V. (2001). Filosofía para hacer las paces. Barcelona: Icaria.

Martínez Guzmán, V. (2018). Educar la imaginación y la creatividad para hacer las paces: El hacer poético. En S. París Albert y S. Herrero Rico (eds.), El quehacer creativo. Un desafío para nuestra cotidianidad (pp. 21-36). Madrid: Dykinson. https://doi.org/10.2307/j.ctv301f3z.5

Max-Neef, M. (2000). Desarrollo a escala humana. Barcelona: Icaria.

Morin, E. (1998). Introducción al pensamiento complejo. Barcelona: Gedisa.

Muñoz Muñoz, F. A. y Molina Rueda, B. (2010). Una cultura de paz compleja y conflictiva. La búsqueda de equilibrios dinámicos. Revista de Paz y Conflictos, 3, 44-61.

Naciones Unidas (2015). Transformar nuestro mundo: La agenda 2030 para el desarrollo sostenible. París: Naciones Unidas.

Ordine, N. (2013). La utilidad de lo inútil. Barcelona: Acantilado.

Ospina-Ramírez, D. A. y Ospina-Alvarado, M. C. (2017). Futuros posibles, el potencial creativo de niñas y niños para la construcción de la paz. Revista Latinoamericana de Ciencias Sociales, Niñez y Juventud, 15(1), 175-192.

Panikkar, R. (1993). Elogio de la sencillez. El arquetipo universal del monje. Pamplona: Editorial Verbo Divino.

París Albert, S. (2015). Repensar los conflictos interculturales y su transformación pacífica desde el paradigma de la complejidad de Heráclito de Efeso. Thémata. Revista de Filosofía, 52, 179197. https://doi.org/10.12795/themata.2015.i52.10

París Albert, S. (2017). Filosofía para hacer las paces con niños y niñas. Un estímulo para la creatividad. Convergencia. Revista de Ciencias Sociales, 75, 65-85. https://doi.org/10.29101/crcs.v0i75.4357

París Albert, S. (2018a). Acciones educativas para afrontar algunos desafíos de la filosofía en el siglo XXI. Daimon. Revista Internacional de Filosofía, 74, 105-119. https://doi.org/10.6018/daimon/267491

París Albert, S. (2018b). Hacia una reconstrucción de las paces creativas para la ciudadanía global. Revista de Paz y Conflictos, 11(1), 159-179. https://doi.org/10.30827/revpaz.v11i1.6461

Pieper, J. (1970). Defensa de la filosofía. Barcelona: Herder.

Sátiro, A. (2011a). La creativitat com a motor de desenvolupament i d'inclusió social. Cantabou, 33, 4-13. 
Sátiro, A. (2011b). Pedagogia per a una ciutadania creativa. Temps d'Educació, 40, 129-144.

Sátiro, A. (2002). ¿Crear? Un artículo para dialogar. En M. Lipman (Ed.), Filosofía y educación (pp. 145-161). Madrid: Ediciones de la Torre.

Sátiro, A. (2005). Cómo el pensamiento vuela cuando jugamos a pensar creativamente. En VVAA (Coords.), Filosofía en la escuela. La práctica de pensar en las aulas (pp. 47-53). Barcelona: Graó.

Sátiro, A. (2009). Creatividad social: necesidad de transformar y crear mundos. En A. Sátiro (Ed.), Pasión por crear, placer de admirar, necesidad de transformar. Iniciación al arte como herramienta de juego, conocimiento y transformación (pp. 96-115). La Coruña: MACUF.

Toro, J. M. (2017). El pulso del cotidiano. Ser. Hacerse. Vivir. Realizarse. Bilbao: Desclee de Brouwer.

Tuvilla Rayo, J. (2004). Cultura de paz y educación. En B. Molina Rueda y F. A. Muñoz (Eds.), Manual de paz y conflictos (pp. 387-425). Granada: Universidad de Granada.

\section{Breve CV de la autora}

\section{Sonia París Albert}

Profesora Contratada Doctora en el área de Filosofía del Departamento de Filosofía y Sociología de la misma universidad. Actualmente, es Directora de la Cátedra UNESCO de Filosofía para la Paz y Coordinadora del Máster Universitario en Estudios Internacionales de Paz, Conflictos y Desarrollo de la UJI. Ha hecho trabajos de investigación en la Universidad de San Francisco (California, EEUU) y ha impartido docencia de postgrado en la Universidad Autónoma del Estado de México (Toluca). Sus principales líneas de investigación son la Historia de la Filosofía, la Filosofía para la Paz y la Transformación Pacífica de los Conflictos, la Filosofía con Niños y la Creatividad. Entre sus últimas publicaciones, destacan: «Filosofía para hacer las paces con niñas y niños. Un estímulo para la creatividad», «El reconocimiento recíproco en la filosofía de Axel Honneth: contribuciones a la transformación pacífica de los conflictos», «Acciones educativas para afrontar algunos desafíos de la filosofía en el siglo XXI». ORCID ID: https://orcid.org/0000-0003-1326-5078. Email: sparis@fis.uji.es 